\title{
In vivo contactless brain stimulation via non-invasive and targeted delivery of magnetoelectric nanoparticles
}

Tyler Nguyen1,2,3, Jianhua Gao1,2, Ping Wang4, Abhignyan Nagesetti4, Peter Andrews2, Sehban Masood2, Zoe Vriesmann2, Ping Liang 5, Sakhrat Khizroev4, Xiaoming Jin $1,2^{*}$

1Indiana Spinal Cord and Brain Injury Research Group, Stark Neuroscience Research Institute \& Department of Anatomy, Cell Biology and Physiology, Indiana University School of Medicine, Indianapolis, Indiana.

2Indiana Spinal Cord and Brain Injury Research Group, Stark Neuroscience Research Institute \& Department of Neurological Surgery, Indiana University School of Medicine, Indianapolis, Indiana.

3Medical Neuroscience program, Indiana University School of Medicine, Indianapolis, Indiana.

4Department of Electrical and Computer Engineering, College of Engineering, University of Miami, Miami, FL.

5Cellular Nanomed Inc., Irvine, CA.

*Corresponding author: Xiaoming Jin, PhD, Indiana Spinal Cord and Brain Injury Research Group, Stark Neuroscience Research Institute \& Department of Anatomy, Cell Biology and Physiology, Indiana University School of Medicine, 320 West 15th Street, NB 500C, Indianapolis, IN 46202. Tel: (317) 278-5766; Fax: (317) 231-0203; Email: xijin@iupui.edu. 


\section{Abstract}

Non-invasive brain stimulation is valuable for studying neural circuits and treating various neurological disorders in humans. However, the current technologies usually have low spatial and temporal precision and poor brain penetration, which greatly limit their application. A new class of nanoparticles known as magneto-electric nanoparticles (MENs) can be navigated to a targeted brain region with a magnetic field and is highly efficient in converting an externally applied magnetic wave into local electric fields for neuronal activity modulation. Here we developed a new method to fabricate MENs of $\mathrm{CoFe}_{2} \mathrm{O}_{4}-\mathrm{BaTiO}_{3}$ core-shell structure that had excellent magneto-electrical coupling properties. Using calcium imaging of organotypic and acute cortical slices from GCaMP6s transgenic mice, we demonstrated their efficacy in reliably evoking neuronal responses with a short latency period. For in vivo non-invasive delivery of MENs to brain, fluorescently labeled MENs were intravenously injected and guided to pass through the blood brain barrier to a targeted brain region by applying a magnetic field gradient. A magnetic field $(\sim 450 \mathrm{Oe}$ at $10 \mathrm{~Hz})$ applied to mouse brain was able to reliably evoke cortical activities, as revealed by in vivo two-photon and mesoscopic imaging of calcium signals at both cellular and global network levels. The effect was further confirmed by the increased number of c-Fos expressing cells after stimulation. Neither brain delivery of MENs nor the subsequent magnetic stimulation caused any significant increases in the numbers of GFAP and IBA1 positive astrocytes and microglia in the brain. This study demonstrates the feasibility of using MENs as a novel efficient and non-invasive technique of contactless deep brain stimulation that may have great potential for translation.

Keywords: Noninvasive brain stimulation, nanoparticles, calcium imaging, two-photon, neuroinflammation. 


\section{Introduction:}

In the last two decades, noninvasive brain stimulation techniques have made important contributions to the understanding of brain neurophysiology and treatment of neurological diseases. The two most common forms of noninvasive brain stimulation used clinically are Transcranial Magnetic Stimulation (TMS) and transcranial Direct Current Stimulation (tDCS) 1, 2, 3. However, they have relatively low spatial and temporal precisions, which greatly diminish their efficacy and application. For example, regular TMS has a spatial resolution of 3-5 cm and depth of penetration of $\sim 1-1.5 \mathrm{~cm}$, which allows for stimulating a cortical gyrus4, 5. tDCS also has very low spatial resolution and limited effects on deep brain structures4, 6, 7. More recent approaches such as optogenetics8, 9, magneto-thermal brain stimulation10, 11, and ultrasound-based transcranial stimulations1212,13,14,14, have improved in ways that were limited in tDCS and TMS (i.e. cellular specificity and spatial and temporal resolutions) but present drawbacks including the requirement of genetic modifications (e. g. optogenetics), invasive brain delivery of particles (e.g. magneto-thermal nanoparticles), and brain tissue damage due to high release of thermal energy (e.g. in focused ultrasound brain stimulation).

The concept of using magneto-electric nanoparticles (MENs) for brain stimulation was first proposed in a theoretical paper by Yue et al in 201215. The same research group led by Sakhrat Khizroev reported the first in vivo MEN-mediated modulation of brain EEG activity by external magnetic field via intravenous injection of MENs in 2015 16. They used coreshell $\mathrm{CoFe}_{2} \mathrm{O}_{4}-\mathrm{BaTiO}_{3} \mathrm{MENs}$ due to their relatively strong magnetoelectric (ME) effect owing to the strain-induced coupling between the magnetostrictive cobalt ferrite cores and piezoelectric barium titanate shells. The nanoparticles can be injected into a vein, forced to cross blood-brain barrier (BBB) and consequently localized to a target region by applying a magnetic field gradient. When exposed to a relatively low magnetic field (100-500 Oe), the MEN's cobalt ferrite ( $\left.\mathrm{CoFe}_{2} \mathrm{O}_{4}\right)$ core experiences non-zero strain due to the magnetostrictive effect. Owing to the ME coupling, this strain propagates through the lattice matched interface to the adjacent barium titanate $\left(\mathrm{BaTiO}_{3}\right)$ shell, which in turn induces a local electric field (on the order of $1000 \mathrm{~V} / \mathrm{m}$ ) due to the piezoelectric effect 16. In this way, magnetic brain stimulation 
using MENs (MENs-MS) can be achieved with a magnetic field intensity that is much lower than what is used for typical TMS $(30,000-50,000 \mathrm{Oe}) 17$. The unique properties of MENs, due to their small size ( $\sim 30 \mathrm{~nm})$ and the ME effect not displayed by any other nanoparticles known to date, may provide significant improvements over currently used techniques in in efficacy, precision, and tissue penetration for noninvasive brain stimulation. However, to date, no direct effect of MENs on stimulating neurons in a focal brain region and/or a large neural network has been demonstrated.

In the current study, we developed a non-invasive technique using MENs to wirelessly stimulate cortical neuronal activity. Using in vitro and in vivo fluorescent and two-photon imaging techniques, we showed that MENs can be drawn to across the BBB and localized to a target cortical region without any apparent signs of neuroinflammation. By activating the MENs with a weak external magnetic field at a specific frequency, we were able to induce cortical activities in individual neurons and in a large neural network in vivo.

\section{Procedures:}

1. MENs synthesis, ultrastructural imaging, and functionalization: MENs were synthesized using the hydrothermal method as described in Guduru et al. 2014 18. Samples for transmission electron microscope (TEM) imaging were prepared by dispersing $1 \mathrm{mg}$ MENs in $2 \mathrm{~mL}$ ethanol and sonicating the mixture for 20 minutes. One drop of the MENs solution was deposited with a pipette on a copper TEM grid substrate. Slowing evaporation at low temperature provided a uniform nanoparticle distribution on the substrate. To eliminate aggregation and potential toxicity, the surfaces of the MENs were functionalized by adding a 2-nm thick coating of glycerol mono-oleate (GMO) as follows: the MENs were washed twice with phosphate-buffered saline (PBS, $\mathrm{pH} 7.4$ ) and sonicated for 2 minutes between washes for uniform dispersion in solution. MENs were then suspended in a GMO solution ( $8 \mu \mathrm{L} \mathrm{GMO} / 1 \mathrm{~g}$ MENs) and mixed thoroughly on a rotator for 12 hours. Excess GMO were then removed by extraction with $1 \mathrm{ml}$ of $70 \%$ ethanol: PBS and two subsequent washes with PBS. Finally, the MENs-GMO 
mixture were suspended in saline and stored at -20 oC for short-term storage of 3-5 days or freeze dried in liquid nitrogen for long term storage for more than 1 week. To prepare fluorescent MENs (fMENs), Texas Red NHS ester was first reacted with oleylamine (2:1 molar ratio) for 18-24 hours on a rotator in a dark environment. Then, hexane was added in drops incrementally to help dissolved the oleylamine-Texas Red mixture. After the solution of oleylamine-Texas Red NHS ester was combined with the GMO-MENs mixture (1:3 volume ratio of GMO-MENs: oleyamine-TexasRed), it was rotated for additional 12 hours to allow binding. Following removal of excess GMO through multiple washes in PBS, the final product was store at $-20 \circ \mathrm{C}$ (short-term storage).

\section{In vitro imaging of cortical neurons in organotypic cultures from GCaMP6s} transgenic mice: All experiments were approved by the Institutional Animal Care and Use Committee (IACUC) of the Indiana University School of Medicine, which are in accordance with National Institutes of Health guidelines for the care and use of laboratory animals. Preparation of cortical slice cultures: Cortical slice cultures were prepared from GCaMP6s transgenic mice that were 3-5 days old $(n=5)$. The animals were anesthetized by rapidly cooling them in ice. After quick decapitation, the brain was removed and immediately immersed in an ice-cold cutting artificial cerebrospinal fluid (aCSF) solution ( $2 \mathrm{mM} \mathrm{KCl}, 1 \mathrm{mM} \mathrm{MgCl}$, 2 $\mathrm{mM} \mathrm{MgSO}_{4}, 1.25 \mathrm{mM} \mathrm{NaH}_{2} \mathrm{PO}_{4}, 26 \mathrm{mM} \mathrm{NaHCO}_{3}, 1$ mM CaCl 2,10 mM DGlucose, 206 mM Sucrose). The cerebellum was then removed, and the brain was glued onto a cutting stage. A vibratome (VT1200, Leica Biosystems, Buffalo Grove, IL.) was used to section the brain into coronal slices of $350 \mu \mathrm{m}$ thick at a cutting velocity of $0.75 \mathrm{~mm} /$ minutes. The slices were then collected and placed in a cold oxygenated aCSF solution (126 mM NaCl, $2.5 \mathrm{mM} \mathrm{KCl,} 2$ mM MgSO 4 , $1.25 \mathrm{mM} \mathrm{NaH}_{2} \mathrm{PO}_{4}, 26 \mathrm{mM} \mathrm{NaHCO}_{3}, 2 \mathrm{mM} \mathrm{CaCl}_{2}, 10 \mathrm{mM}$ D-Glucose). Intact slices were then selected and arranged carefully on Millipore Millicell culturing inserts (Millipore Sigma, St. Louis, MO). The slices were cultivated at $\sim 37 \circ \mathrm{C}$ in $95 \% \mathrm{O}_{2} / 5 \% \mathrm{CO}_{2}$ in a culturing medium consisting of $46 \%$ Basal Medium Eagle (BME), 25\% Earle's Salt Solution, 25\% fetal bovine solution (FBS), $3 \%$ of $22 \%$ 
Glucose solution, and 1\% L-Glutamine-Penicillin-Streptomycin solution. 75\% of the medium was changed with new medium every three days.

Magnetic field application: Prior to imaging, a cultured brain slice was removed from the culturing insert by cutting the membrane surrounding the slice and placed in a custom-made recording chamber filled with $37 \circ \mathrm{C}$ aCSF. The recording chamber was a $2-\mathrm{cm}$ diameter well with a glass bottom that was filled with aCSF containing $5 \mu \mathrm{L}$ of MENs (at a final concentration of $5 \mathrm{mg} / \mathrm{mL}$ ). A 5000 Oe conical magnet was used to apply a strongly localized magnetic field to the bottom of the chamber for approximately 15 minutes to draw the MENs to a focal area of the slice. For magnetic stimulation, two electromagnets were put on opposite sides of the recording chamber in a Helmhotz pair arrangement according wherein the diameter of each coils was comparable to the separation between the coils. This arrangement was chosen to significantly reduce or eliminate a magnetic force to ensure the nanoparticles did not physically move during the stimulation process. A sinusoidal magnetic field of approximately 450 Oe was applied in a pulsed mode with 50 ms pulse width at $10 \mathrm{~Hz}$ to stimulate the MENs during the imaging process.

Two-photon imaging: Two-photon imaging of cortical slices was performed using a Prairie Technologies Ultima 4423 two-photon system (Bruker Inc., Middleton, WI) equipped with a MaiTai Ti: Sapphire laser (Newport, Mountain View, CA) tuned to $900 \mathrm{~nm}$. Band-pass filtered fluorescence $(560-600 \mathrm{~nm})$ was collected by photomultiplier tubes of the system. The average laser power on the sample was 20-30 mW. All images were acquired at a resolution of $512 \times 512$ pixels using a 20x water-immersion objective (Nikon Instruments Inc., Melville, NY). Images were capture at a rate of 4-5 fps. Typically, each recording session consisted of 30 seconds of baseline recording, 40s with magnetic field application, and 30 seconds of post-magnetic application period.

\section{Ex vivo calcium imaging of acute GCaMP6s cortical slices: Cortical slice} preparation: Thy1-GCaMP6s transgenic mice, in which layers I//II and V pyramidal neurons express GCaMP6s calcium sensor protein, were purchased 
from the Jackson's Laboratory and bred locally. Adult mice $(n=4)$ were anesthetized with $78 \mathrm{mg} / \mathrm{kg}$ ketamine and $22 \mathrm{mg} / \mathrm{kg}$ xylazine (i. p.) and decapitated. The brain was removed and immediately placed in an ice-cold oxygenated sucrose artificial cerebral spinal fluid (s-ACSF) solution (206 mM sucrose, 2 mM KCl, 1 mM MgCl2, 2 mM MgSO4, 1.25 mM NaH2PO4, 26 mM NaHCO3, $10 \mathrm{mM}$ D-glucose, $1 \mathrm{mM} \mathrm{CaCl}$ ). After 1-2 minutes, the brain was situated on a cutting stage with the cortex facing the approaching blade. Slices of $350 \mu \mathrm{m}$ thickness were cut with a Vibratome (Leica VT1200S; Leica, Nusslock, Germany) while the brain was submerged in the cutting solution. The slices were then incubated at 37 。C for 1 hour in a chamber filled with oxygenated ACSF (124 mM NaCl, 3 mM KCl, 2 mM MgSO4, 1.25 mM NaH2PO4, 26 mM NaHCO3, $10 \mathrm{mM}$ D-glucose, $1 \mathrm{mM} \mathrm{CaCl} 2)$. Calcium imaging: A recording chamber was made by attaching a temperature control heated well (Thermal Well Temperature Controller TC-100, BioScience Tools, San Diego, CA) onto a coverslip. Liquid inflow and vacuum outflow tubing were installed to generate ACSF current through the chamber. A cortical slice was placed in the chamber and secured with a metal ring. Calcium activities of the slices was imaged with a system consisting of a Leica DM6000 FS upright microscope with a fluorescence light source directed through a 10x water immersion objective (Leica, Nusslock, Germany). Images were capture with an iXON EMCCD DU-88U camera system (Andor USA, Concord, MA) at $\sim 45-50$ fps. Cortical slices were imaged at 35 oC in aCSF with $20 \mu \mathrm{M}$ bicuculline.

MENs loading and stimulation parameters: After the liquid flow of the recording chamber was turned off briefly, $20 \mu \mathrm{L}$ of MENs (concentration of $5 \mathrm{mg} / \mathrm{mL}$ in aCSF) was added to the chamber. A 5000 Oe conical magnet was applied under the glass for $\sim 5-8$ minutes to draw MENs to a small area of the slice. Liquid flow was turned back on prior to stimulation and recording. For stimulation, the recording chamber was placed between a pair of electromagnets. A unipolar magnetic field of 750-875 Oe with 200 ms pulse-width was applied during the imaging process. 
3. In vivo two-photon imaging of fMENs in cerebral blood stream: Thy1GCaMP6s mice $(n=5)$ were anesthetized with Ketamine/Xylazine mixture (87.3 $\mathrm{mg} / \mathrm{kg} / 13.7 \mathrm{mg} / \mathrm{kg}$ ) and the scalp was removed. A cranial window ( $3 \mathrm{~mm}$ in diameter) was made in an area $1.5 \mathrm{~mm}$ lateral from the midline and $1 \mathrm{~mm}$ posterior from the bregma. Two pieces of cover glass (a 3-mm diameter glass adhered to a 5-mm diameter glass) were glued together with cyanoacrylate glue with the 3-mm glass facing the brain surface. After the glass was installed on the cranial window, an L-shaped titanium head-plate was glued on the posterior region of the head for head fixation during imaging. After the animals were allowed to recover for 7 to 10 days, a single injection of $200 \mu \mathrm{l}$ of fMENs at 200 $\mu \mathrm{g} / \mathrm{ml}$ was made through the retro-orbital route. Two-photon images of cerebral blood vessels were taken at baseline, and $0,5,10,20$, and 30 minutes after fMENs injection.

In vivo two-photon imaging after fMENs delivery: In GCaMP6s mice with cranial windows prepared as described above, an injection of $200 \mu \mathrm{L}$ fMENs at 5 $\mathrm{mg} / \mathrm{ml}$ was made through the retro-orbital route. After two sets of stacked cylindrical magnets of 6000 Oe field strength were separately placed on top of the cranial window and beneath the mouth of the mouse for 10 minutes, a conical magnet with the field of 5000 Oe at the tip was applied to the cranial window to further localize the MENs. Images were taken before and after fMENs injection, after fMENs injection with magnet application, and 24 hours after MEN delivery.

\section{In vivo two-photon calcium imaging of cortical neurons in GCaMP6s}

transgenic mice: Mice with cranial windows prepared as described above were used in this experiment. After the animals were sedated via $4 \%$ isofluorane and maintained with intraperitoneal injection of chloprothixene $(0.04 \mathrm{mg} / \mathrm{ml})$, they were injected retro-orbitally with $200 \mu \mathrm{lMENs}$ at $200 \mu \mathrm{g} / \mathrm{ml}$. Following the delivery of the MENs to cortex using the same technique described above, the animal's head was stabilized by fixing the L-shaped titanium metal plate to a custom-made base and the body temperature was maintained. Magnetic stimulation was made by placing two electromagnets ( $500 \mathrm{~ms}$ pulse-width at $\sim 300-450$ Oe) closely on both side of the head. Again, the Helmholtz pair 
arrangement of the electromagnet was chosen to minimize the pulling magnetic force on the nanoparticles during the stimulation events. Two-photon images of calcium transients of layer II/III neurons were taken at baseline and during and after magnetic stimulation. In each imaging field, two optical planes in layer II/III were imaged for 2 minutes at 4-5 frames per second (fps). We tested a combination of different stimulation frequencies $(5,10,20,50$, and $100 \mathrm{~Hz})$.

\section{Mesoscopic calcium imaging of cortical activity in vivo: Thy1-GCaMP6s} transgenic mice were anesthetized with Ketamine/Xylazine mixture $(87.3 \mathrm{mg} / \mathrm{kg} /$ $13.7 \mathrm{mg} / \mathrm{kg}$ ) and the scalp was removed. After a large cranial window (approximately $10 \times 8 \mathrm{~mm}$ ) was made by removing the skull, a piece of curved cover glass was installed and tightly sealed with super glue and dental cement. The animals were allowed to recover for 7 to 10 days prior to imaging. A mesoscopic imaging set-up was built similar to previously described method $19,20$. Images were captured with a two-lens system composed of a top zoom Nikkor 70-300 mm lens of aperture f3.5-5.6 set at 70mm at f3.5 coupled with an inverted lens adapter to a bottom Nikkor $50 \mathrm{~mm}$ prime lens at f1.4. Videos were captured with an iXON EMCCD DU-88U camera system (Andor USA, Concord, MA) controlled by MetaMorph software. Delivery of MENs and magnetic stimulation paradigm were done exactly as described in two-photon imaging.

6. Data analysis of Ca imaging: Two-photon imaging data were analyzed post hoc using ImageJ software (NIH) with a Time Series Analyzer plugin (Balaji J. UCLA). All active cells (cells that produce calcium fluorescence flashes throughout all frames) within each frame were analyzed. To analyze individual cells, three separate background points were chosen by drawing circles with the same diameter as the respective cell body in the surrounding area. The background areas selected did not include dendrites and other visible neuronal structures. Data obtained included signals of cell body and three background fluorescent profiles. For mesoscopic brain imaging, a circular area of interest ( $1 \mathrm{~mm}$ in diameter) was selected, with an adjacent blood vessel selected as background to compensate for changes in general brightness. The fluorescence calcium signal 
of a cell $\Delta F / F$ was calculated by equation (1) excerpt from Chen et al. 201321 that was developed by Kerlin et al, 201022:

$$
\frac{\Delta \boldsymbol{F}}{\mathbf{F}}=\frac{\left(\boldsymbol{F}_{\text {true }}-\boldsymbol{F}_{\boldsymbol{B}}\right)}{\boldsymbol{F}_{\boldsymbol{B}}} \quad \text { and } F_{\text {true }}=F_{\text {cell }}-r * F_{B}
$$

where $F_{B}$ is the average baseline fluorescence over three different regions surrounding the cell of interest and $r$ is the contamination ratio standard constant 0.7. The data were then used to construct a calcium peak profiles using OriginPro 9.1 (OriginLab, Northampton, MA). Analyses of peak amplitude, frequency, and durations were done with Peak analysis toolbox of OriginPro 9.1.

7. C-fos staining of cortical neurons: C57BL6J mice ( $\mathrm{n}=4$ animals/group) were assigned into three groups: control (saline injection + MS $(5 \times 2$ minutes MS at 10 $\mathrm{Hz})$ ), MENs (MENs delivery without magnetic stimulation), and MENs-MS (MENs delivery and MS $(5 \times 2$ minutes MS at $10 \mathrm{~Hz}))$. MENs delivery and magnetic stimulation (MS) were done similarly as described above.

Brain tissues of these mice were stained for c-Fos protein, an indicator of action potential firing23. Four hours after the treatment, the mice were deeply anesthetized and perfused transcardially with PBS buffer, followed by $4 \%$ formaldehyde PFA at room temperature. The brains were then dissected and placed in $30 \%$ sucrose for $48-72$ hours at $4 \circ \mathrm{C}$. The tissue was then frozen and sectioned with a cryostat. Immunofluorescence was performed on free-floating sections by incubating overnight with a primary antibody for c-Fos (rabbit; $1: 800$, Sigma Aldrich G3893) and followed by incubation with fluorescent goat antirabbit secondary antibody (1:200; Invitrogen, Carlsbad, CA). For nuclear staining, 4', 6-diamidino-2-phenylindole (DAPI; 1: 10,000) was added to the solution for a final 5 min. Finally, slices were imaged using a Neurolucida imaging system.

8. Immunofluorescence analysis of astrocytes and microglia: To determine potential glial reactivity and neuroinflammation induced by the MENs, we used an antibody against ionized calcium binding adaptor protein (IBA1) to label microglia, and an antibody against Glial Fibrillary Acidic Protein (GFAP) to label astrocytes24, 25. C57BL6J mice were divided into three groups: control (saline 
+MS), MENs (MENs without MS), and MENs-MS (MENs delivery with MS). The MENs delivery and MS paradigm were identical to the c-Fos experiment described above. Within the MENs-MS group, subgroups of mice were euthanized at 4 hours, 24 hours, 3 days, or 1 week after MS. Mice were perfused and brain slices were stained as described above. Anti-GFAP (mouse; 1:800, Sigma Aldrich G3893) and anti-IBA1 (goat; 1:200, ABCam ab5076) antibodies were followed by secondary antibodies of goat anti-mouse Cy5 (1:500, Jackson Immuno) and donkey anti-goat 488nm (1:1000, Fischer Scientific).

9. Statistics: Mean values and final plots were developed in Microsoft Excel, Jmp Analysis 11 (SAS Institute Inc. 2013. Cary, NC), and GraphPad Prism 6 (GraphPad Software, La Jolla, California). All statistical analyses were done with Jmp Analysis 11 and GraphPad Prism 6. ANOVA analyses were used for the following comparisons: calcium amplitudes of the same group across different time points (Repeated measures Anova), amplitudes and frequencies of calcium imaging data (one-way), calcium amplitudes and frequencies across different tested magnetic frequencies and different cortical region in mesoscopic imaging (one-way), and compare of positive stained cells between groups of c-Fos, IBA1, and GFAP immunohistochemistry experiment (one-way). For comparisons that yielded statistical significances, Tukey's HSD post-hoc analyses were applied for further comparisons between specific groups.

\section{Results}

\section{MENs synthesis and characterization}

We developed MENs with a true coreshell nanostructure, in which the magnetic core was intrinsically coupled to the piezoelectric shell via perfect lattice matching (Figure 1). The size of the MENs was on the order of $30 \mathrm{~nm}$. With this new synthesis, we were able to develop MENs with a high ME coefficient of $>5 \mathrm{~V} / \mathrm{cm} / \mathrm{Oe}$ in the frequency range, 0 to $200 \mathrm{~Hz}$, under study (data not shown). 


\section{MENs-MS induced neuronal activity in cultured and acute cortical slices}

We first assessed the effects of MENs-MS on neuronal activity in organotypic cortical slices prepared from GCaMP6 transgenic mice. After the slices were cultured for 5 days old in vitro, they were loaded with MENs and stimulated with a magnetic field (10 Hz sinusoidal wave at 450 Oe for $10 \mathrm{~s})$. The peak amplitude of calcium transients from individual neurons increased nearly $200 \%$ (Figure $2 \mathrm{~A}$ and $\mathrm{B}, \mathrm{F} / \mathrm{F}_{\circ} 0.809 \pm 0.12$ for baseline vs $3.586 \pm 0.78$ for magnet-on, $p<0.005$, Repeated-measures ANOVA, Tukey's HSD). In neurons that were initially active, we saw a significant increase in calcium signals, whereas in neurons that were not active, the magnetic stimulation induced calcium activity (Figure 2A and B). We also found a significant increase in spike frequency during the period when the MS was turned on (Figure $2 \mathrm{C}$ and $\mathrm{D}, 1.43$ $\pm 0.13,2.6 \pm 0.21$, and $1.46 \pm 0.17$ spikes/cell for baseline, MS-on, and MS-off periods, respectively, $p<0.01$ for comparison between baseline and MS-on, Repeated ANOVA, Tukey's HSD).

In order to improve the temporal precision of the stimulation, we used a single pulse (unipolar square pulse at 750-875 Oe, $200 \mathrm{~ms}$ duration) of magnetic field and tested the effect in acutely prepared cortical slices from GCaMP6 transgenic mice (Figure $3 \mathrm{~A}$ ). The mean amplitude of calcium spikes at baseline was $0.127 \pm 0.035$, which became $0.541 \pm 0.056$ during magnetic stimulation, and returned to $0.121 \pm 0.32$ after the magnet was turned off $(p<0.005$, Repeated ANOVA, Tukey's HSD; Figure 3B). Most of the evoked calcium responses had a shorter latency period (Figure $3 \mathrm{C}$ ), with over $80 \%$ of the responses occurring in less than 1 second and $32 \%$ of the responses in less than 150 milliseconds after the magnetic pulse was turned on.

\section{Intravenous injection of MENs followed by magnetic delivery to brain}

We first use repeated in vivo two-photon imaging of cerebral blood circulation to determine how long intravenously injected MENs would be detectable in blood stream. Immediately after injecting fMENs into the tail vein of 
a mouse, we found many fluorescent particles in blood stream of cortical vessels. These particles decreased rapidly over a short period of time (Figure 4A-B): with the fluorescence intensity in blood stream dropping to about a half in 15 minutes and to almost zero in 30 minutes after injection (Figure 4B). This drop-in particle number suggested that the optimal time window for applying a magnet for MENs delivery is within $\sim 10$ minutes after intravenous injection of MENs.

We used fMENs to assess the effectiveness of applying permanent magnet for delivering MENs into brain parenchyma. Following intravenous injection of fMENs and application of a conical magnet on mouse head for 20 minutes (Figure 4C-D), in vivo two-photon imaging showed that fluorescence signals were visible through cranial windows in mice with fMENs delivery, but not in mice with MENs injection only. The result suggests that the fMENs were drawn by a magnetic field to cross BBB and entered brain parenchyma in vivo (Figure 4C-D). When the same mice were imaged again 24 hours later, significant signals of MENs were still evident in the same region (Figure 4D), suggesting successful delivery of MENs to cortical tissue.

\section{MENs-MS induced cortical neuronal activity and network activity in vivo}

We used in vivo two-photon imaging in GCaMP6 transgenic mice to assess whether MENs-MS would evoke cortical neuronal activity (Figures 5A-D). Following delivery of MENs and MS with a $10 \mathrm{~Hz}$ magnetic wave, we found that there was a dramatic increase in the mean amplitude of calcium spikes (Figure 5C. F/Fo of $1.50 \pm 0.09,2.37 \pm 0.18$, and 1.36 for baseline, MS-on, and MS-off, respectively, $p<0.05$, Repeated-measures ANOVA, Tukey's HSD), and spike frequency $(3.08 \pm 0.27$ spikes/cell vs. $1.96 \pm 0.24$ for baseline with $p=0059$ and $1.54 \pm 0.25$ for magnet off with $\mathrm{p}<0.05$, repeated ANOVA, Tukey's HSD; Figure 5D) in cortical layer II/III neurons. The efficacies of magnetic waves at 5, 10, 20, 50 , and $100 \mathrm{~Hz}$ on activating cortical neurons were also tested in vivo. We found that, for every magnetic frequency applied during the stimulation period, a significant increase in somatic calcium spike amplitude occurred (Figure 5E). The 
strongest neuronal responses (i.e. the highest average spike amplitude and frequency) were evoked by magnetic waves at 5, 10 and $20 \mathrm{~Hz}$ (Figure 5E and F). Therefore, the $10 \mathrm{~Hz}$ frequency was picked as the standard for in vivo stimulation in most of the experiments of our study.

Multiple brain stimulations over a time period may be required to achieve treatment effect in certain neurological diseases. To evaluate this, we gave animals a single MENs delivery, and then performed MS and calcium imaging at different time points (Figure 6). We found a large increase in the amplitude of calcium spikes when MS is applied immediately after MENs delivery, when compared with baseline where ms was applied without MENs treatment (mean F/Fo of $2.7 \pm 0.27$ vs $1.13 \pm 0.07$ of baseline, $p<0.05$, one-way ANOVA, Tukey's HSD, Figure 6B, D). Neuronal activity could still be stimulated with similar parameters of MS at 24 hours after MENs delivery $\left(F / F_{\circ}\right.$ of $2.35 \pm 0.28, p<0.05$, one-way ANOVA, Tukey's HSD), but not at 72 hours after (Figure 6D. F/Fo of $1.21 \pm 0.18, p>0.05$, one-way ANOVA). We also found a higher number of active cells after MENs treatment up to 24 hours ( 90 cells of MENs+MS and 82 cells of 24 hours vs. 62 of ms-only baseline, Figure $6 \mathrm{C}$ ), and recover to baseline level at 72 hours ( $~ 55$ cells, Figure $6 \mathrm{C}$ ). The result suggests that a single delivery of MENs to brain is effective for non-invasive brain stimulation for at least 24 hours.

To assess the effect of MENs-MS on global network activity, we applied mesoscopic brain imaging technique to evaluate hemispheric calcium response in GCaMP6 transgenic mice (Figure 7A-F). The epicenter region was defined as the location where a permanent magnet was applied to draw MENs to a local cortical region (Figure 7A). Application of a $10 \mathrm{~Hz}$ magnetic field induced cortical calcium spikes with significantly higher amplitude and varying latencies in the epicenter and contralateral cortex ( $p<0.05$, repeated ANOVA, Tukey's HSD, Figure $7 \mathrm{~B}, \mathrm{C})$. Comparing the responses among the epicenter and different ipsilateral and contralateral cortical regions revealed a highest peak amplitude at the epicenter and gradual reduction in amplitude $(p<0.05$, one-way ANOVA, Tukey's HSD, Figure 7D, E, and F) and an increase in the latency period (Figure 7D, G, and H) at locations further away from the epicenter. The results suggest 
that neuronal activity was initiated at the epicenter where MENs are localized and spread to more distal brain regions and the contralateral cortex. These data provide strong evidence that MENs-MS can activate and enhance neuronal activity in vivo.

\section{MENs-MS increased cortical c-Fos expression}

We found an increase in cortical c-Fos expression only for animals that has both MENs delivery and MS, with an average of $176 \pm 27.5$ cells/counted region compare to $70.5 \pm 15.25$ for MENs only group and $51 \pm 8$ for MS-only group $(p<0.05$, one-way ANOVA, Tukey's HSD, Figure 8A, B). MENs only group and MS-only group had similar densities of c-Fos positive cells (Figure 8A, B). The results further support that MENs-MS induced cortical neuronal activity in vivo.

6. MENs-MS did not induce astrogliosis and microglial activation: Delivering MENs to a specific brain region may initiate inflammatory responses of glial cells, particularly astrocytes and microglia (Figure 9A-H). To assess whether astrogliosis and microglial activations occurred, we made intravenous injection of $200 \mu \mathrm{l}$ of MENs at $200 \mu \mathrm{g} / \mathrm{ml}$ and magnetic delivery of MENs. Mice that received MENs delivery and MS were also assessed at various time point after the stimulation. We observed no significant differences in the densities of both IBA1 and GFAP positive cells among all the groups (Figure $9 \mathrm{~A}-\mathrm{C}, \mathrm{G}, \mathrm{H}$ ). Considering these results together, MENs-MS did not induce any apparent inflammatory response in mice.

\section{Discussion:}

In the present study, we investigated the effects of MENs-MS for non-invasive activation of cortical neurons in vitro and in vivo. By applying an external magnetic field, we non-invasively delivered MENs across the BBB to the brain and wirelessly stimulated neuronal activity with a short latency in a focal cortical region with great 
efficiency and reliability, without causing detectable neuroinflammatory reaction in the brain.

We developed MENs with a true coreshell nanostructure in which the magnetic core is intrinsically coupled to the piezoelectric shell via perfect lattice matching (Figure 1). To the best of our knowledge, such nanostructures have been fabricated for the first time. Enabling such matching is the key to maximizing the ME coefficient, which in turn is the most important feature of these nanoparticles enabling the creation of an efficient nanotransducer to activate neurons. The small size, on the order of $30 \mathrm{~nm}$, is important not only for their easy penetration through the blood-brain barrier (BBB) but also for ensuring that these nanoparticles can be precisely placed close or directly on the neuronal membrane for maximizing their stimulating capabilities.

Because magnetic stimulation using a pair of electromagnets generates a high level of electromagnetic noise, we used calcium imaging techniques to record neuronal activities instead of using electrophysiological recording. Calcium imaging allowed us to efficiently examine neuronal activities not only in individual neurons in vivo and in vitro but also in both cortical hemispheres. Upon magnetic application, we observed dramatic increases in neuronal calcium response after a short latency, with about one third of responses having a latency period of <150 ms. Since calcium imaging signals are inherently limited by its slower kinetics than electrical signals and GCaMP6s signal is known to have a temporal resolution of $100-150$ ms21, the actual latency is estimated to be $50 \mathrm{~ms}$ and less. This is a good temporal resolution and a clear indication of the stimulatory effects of MENs on neuronal activity. There were significant increases in both calcium transient amplitude and spike number only during the period when the magnet was turned on. Furthermore, the increases in calcium transient amplitude were seen at all the tested frequencies of magnetic stimulation. Calcium response frequency also elevated significantly, where the most dramatic gains were seen between 5 and 20 $\mathrm{Hz}$ of magnet waves.

For in vivo brain stimulation, we first demonstrated that the MENs can be noninvasively delivered to brain parenchyma through intravenous injection followed by application of a permanent magnet on a target region of the skull. The transient 
existence of fMENs in the blood stream for less than 30 minutes suggests that an effective time window for magnetic delivery of MENs lasted only for less than 30 minutes. We found that the MENs could be drawn across the BBB and localized to a region of the cortical hemisphere where the magnet is applied on the skull. The presence of MENs remained in the brain tissue for at least 24 hours after the delivery. Earlier reports have shown that these MENs, after magnetic delivery to the brain, appear to be localized mainly in the extracellular space both in CSF26 and binding to extracellular membranes of various type of CNS residential cells 16, 26. However, MENs are also found to be distributed in cell cytosol26. The chemical structure and integrity of MENs were not compromised during the process of navigation from the blood stream across the endothelial cell layers of the BBB to be localized in the brain26. We also assessed the longitudinal profile of MENs effects on neuronal activity and found their stimulatory effects lasted for at least one day after a single treatment and dissipated within three days. This result suggests the majority of the delivered MENs are cleared from the brain within three days, which may reduce potential side-effects or toxicity of the MEN. However, to achieve repetitive brain stimulation over a longer period, additional MEN delivery or modification of the MENs will be required to improve their density or retention. The whole brain mesoscopic imaging revealed activity changes in a spatially dependent manner relative to the location of MENs, suggesting that targeted stimulation of a brain region can be achieved by delivering MENs to a focal brain region defined by the strength of a magnetic field gradient.

One of the major hurdles in nanoparticle-based treatment techniques is the potential toxicity in vivo due to nanoparticle agglomerations (e.g. aggregations). Nanoparticle agglomerations could induce severe toxicity and inflammation that may lead to abnormal gene expression, tissue degeneration, and cell death27, 28, 29. To prevent particle agglomerations, we coated the surface of MENs with a waterdispersible glycerol mono-oleate (GMO), a well-known water-insoluble and neutral stabilizer that helps prevent aggregates and increases membrane crossing capability 30 , 31. We assessed the neuro-inflammatory response to MENs by evaluating microglial and astrocytic activations at multiple times point after MEN delivery and found no significant changes in microglial activation nor astrogliosis up to one week after MENs delivery and 
magnetic stimulation. In addition to our findings, Kaushkik et al. also reported no changes in peripheral immune response, liver and kidney toxicity, and motor behavior up to one week after delivering MENs to the cerebral cortex26. Furthermore, the MENs have been shown to produce no detectable toxicity on human astrocyte and peripheral blood mononuclear cells at a concentration of $0-200 \mu \mathrm{g} / \mathrm{ml}(\sim 10 \mathrm{ug} / \mathrm{kg} \mathrm{MENs})$, which was sufficient to effectively induce an EEG response16. Particle functionalization via external coating, such as the $\mathrm{SiO}_{2}$ coated - CoFe2O4-PVP (polyvinyl-pyrolidone), was shown to have no toxicity to any vital organs whether it is tissue damage or chromosomal damage32. Taken together, these results provide encouraging information on the safety of MENs to the body and brain in rodents and supports its potential application for translational research and clinical treatment.

In addition to imaging neuronal activity, we also stained mice cortical brain slices for c-Fos expression, a classical indicator of activity (e.g. action potential firing)23, to confirm the stimulatory effect of MENs on neuronal activity. By comparing sham and MENs-delivery only animals, we found a significant increase in the number of cortical cFos expressing cells in MENs-MS animals. Although c-Fos expression in CNS is also observed in some activated astrocytes in inflammatory disease models33, 34, our result of no increase in the number of activated astrocytes (Figure 9) suggests that the increased number of c-Fos positive cells are likely activated neurons, which provides histological evidence supporting the effect of MENs-MS on activating neurons. Taken together, these results demonstrate a ground-breaking ability of MENs to enable wireless control of neuronal activity.

Our goal of this study was to demonstrate as a proof-of-concept the ability of MENs to be localized in the brain and efficiently evoke neuronal activity. To further exploit the potential of MENs, additional effort is needed to precisely deliver MENs to a focal region of the brain and to achieve cell-type specific activation. One approach would be to conjugate MENs with an antibody against a cell membrane protein to enable attachment of MENs to a specific cell type or even a subcellular compartment. This approach may allow precise delivery of MENs to excitatory or inhibitory neuronal population. For the past two decades, there have been efforts to enhance delivery of substances, including metal-based magnetic nanoparticles, across the BBB 35, 36, 37. 
One popular way that has been used widely both in research and in clinical application is pre-treatment with BBB-opening compounds such as mannitol 38 and borneols9. Combining these two approaches will help increase MENs delivery to the brain in a more specific manner.

In conclusion, this study demonstrates the ability of using MENs to wirelessly activate individual cortical neurons and cortical network in vitro and in vivo. We have shown that MENs can be forced to penetrate the BBB to enter brain parenchyma under a magnetic field and we have defined the effective time window and parameters for the delivery. Our in vitro and in vivo calcium imaging data support that MENs-MS evoke cortical neuronal activity with fast temporal resolution at cellular and global network levels. Furthermore, the process of MENs delivery and magnetic stimulation did not induce detectable neuroinflammation. In contrast to current non-invasive brain stimulation techniques, brain stimulation based on MENs offers a contactless, reliable, and efficient approach to modulate brain activity without the need of genetic modification. With further optimization in MENs delivery and cell- and region-specific targeting, this technique could potentially open a new door to a more robust and precise brain control that currently is not possible. 


\section{Acknowledgements}

This publication was made possible with partial support from the N3 program of the DARPA of the Department of Defense (SK, XJ, and LP), the National Science Foundation (NSF) under the grant number ECCS-1935841 (SK and XJ), and from the pre-doctoral fellowship to TN of National Institute of Health grant number $\mathrm{NIH}$ UL1TR002529 (A. Shekhar, PI), National Center for Advancing Translational Sciences, Clinical and Translational Sciences Award and the Indiana University Department of Medicine.

\section{Author Disclosure Statement}

The authors declare that the research was conducted in the absence of any commercial or financial relationships that could be construed as a potential conflict of interest. 


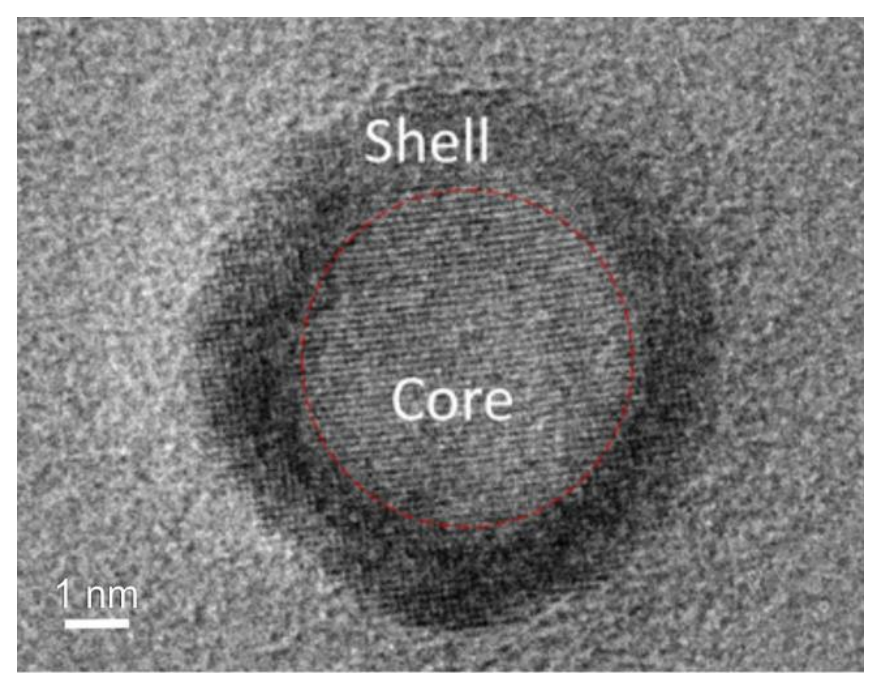

Figure 1. Transmission electron microscopy (TEM) image of a magnetoelectric nanoparticle (MEN). A TEM image demonstrates an almost-perfect lattice matched surface interface between the magnetostrictive core and the piezoelectric shell (shown with broken line) of the coreshell nanostructure. 


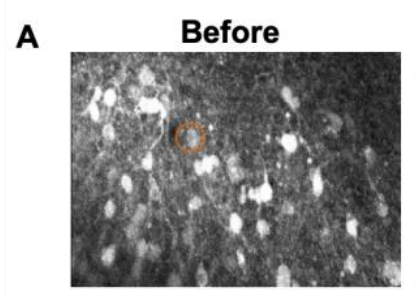

C

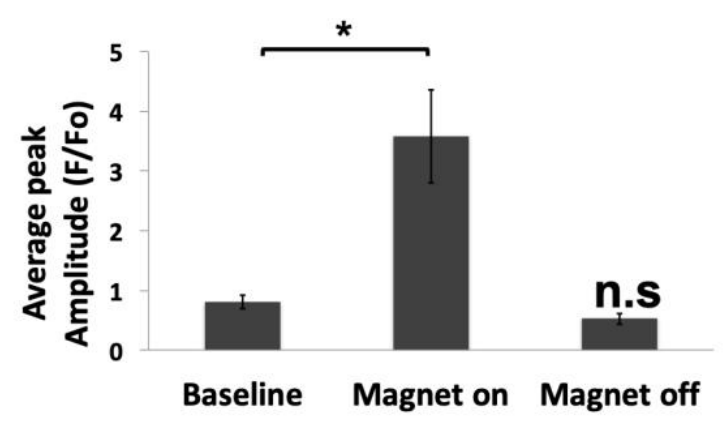

During
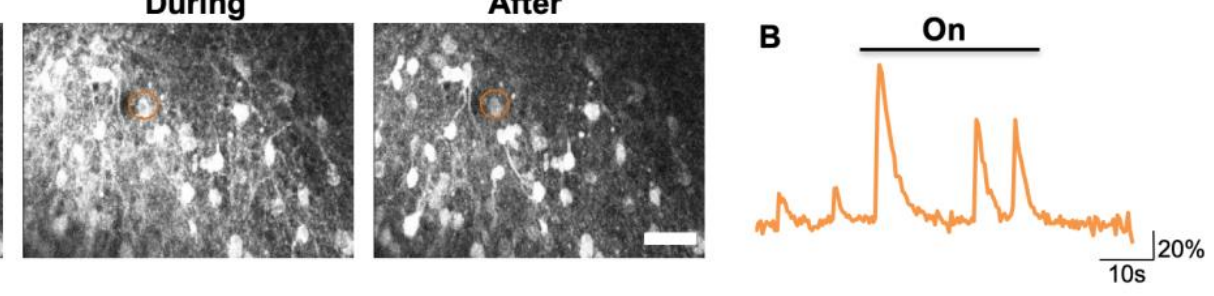

D

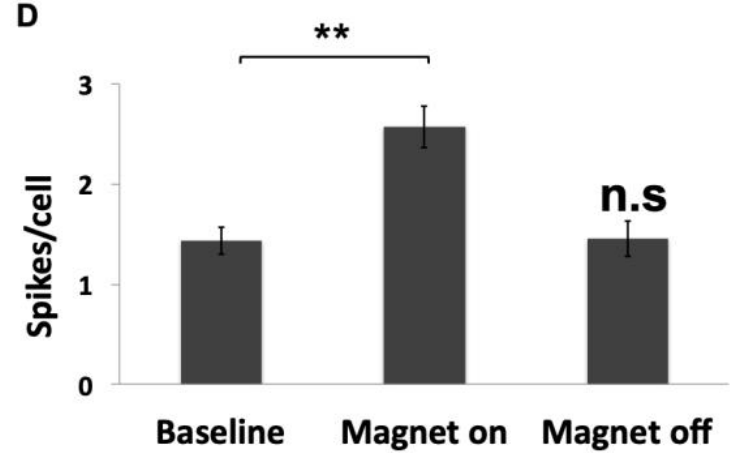

Figure 2. Magnetic stimulation of MENs-loaded cortical slice cultures induced neuronal activity. Cultured cortical slices that expressed GCaMP6 in pyramidal neurons were loaded with MENS by adding $20 \mu \mathrm{l}$ of $5 \mathrm{mg} / \mathrm{mL}$ MENs solution, followed by applying a conical permanent Neodymium (Nd) magnet for $\sim 15$ minutes. Magnetic stimulation was made with a pair of electromagnets at $10 \mathrm{~Hz}$. A. Representative twophoton images of calcium signals in periods before, during, and after magnetic stimulation. B. A sample trace of calcium transients measured from a neuron labeled with red circles in (A). C, D. Increases in average calcium spike amplitude (C) and number of spikes per cell (D) during the stimulation period. Calcium activity returned to baseline level after the magnet was off. Scale bar: $50 \mu \mathrm{m} .{ }^{*}: p<0.05,{ }^{* *}: p<0.01$, Repeated measured ANOVA, Tukey's HSD. $n=9$ slices. 


\section{A}
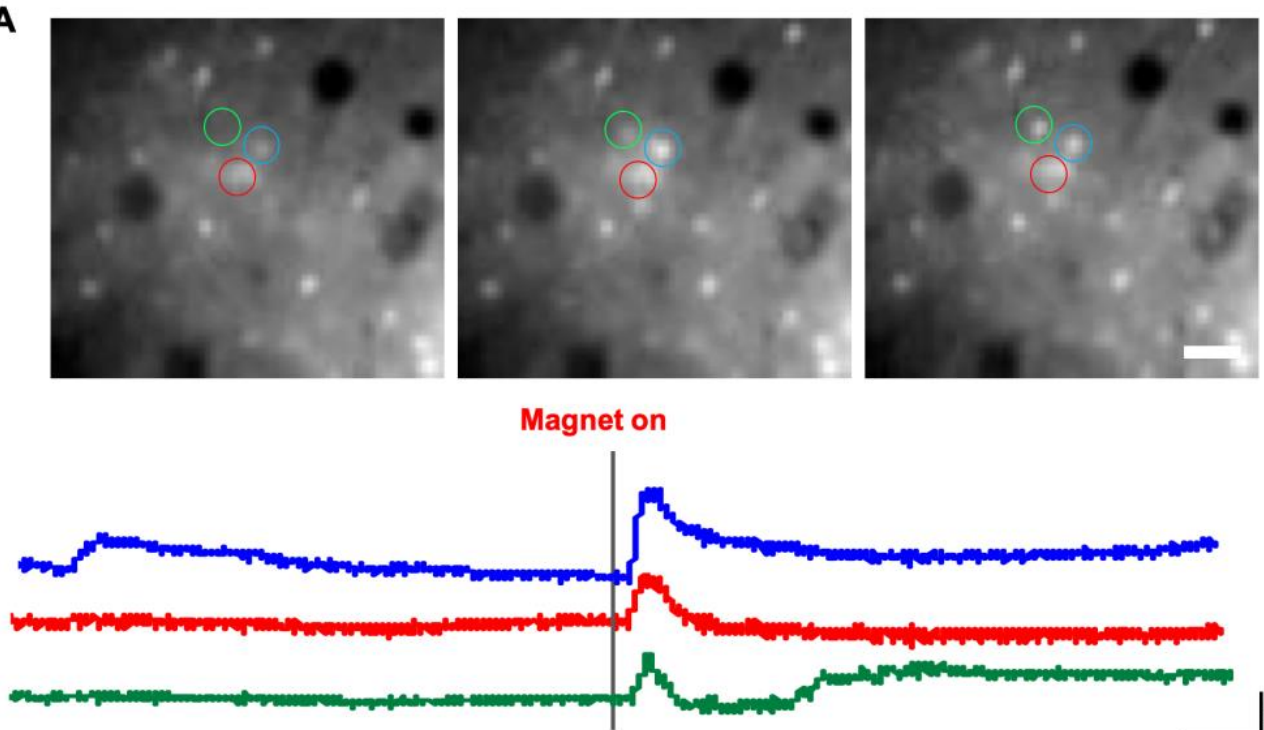

$20 \%$

B

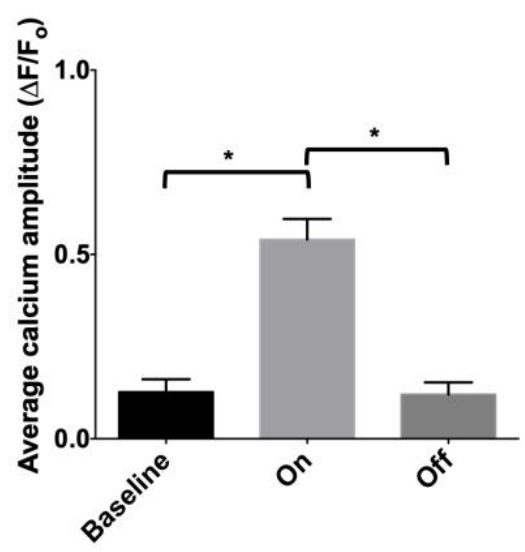

C

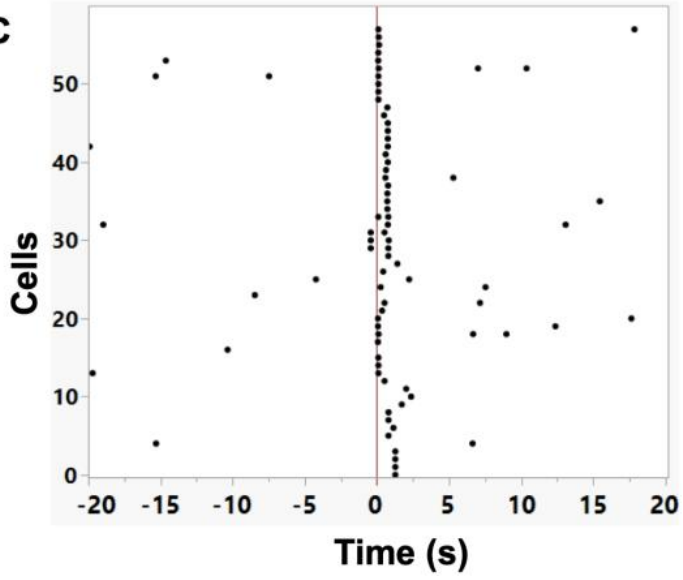

Figure 3. Single unipolar magnetic pulses induced neuronal activity in MENsloaded cortical slices. Cortical slices prepared from GCaMP6 transgenic mice were loaded with MENs similar to Fig. 2 and stimulated with single pulses of magnetic field ( 750-875 Oe at $200 \mathrm{~ms}$ pulse-width). A. Sample images of neurons in a slice and traces of calcium transients measured from the circled neurons. B. Average peak amplitudes of calcium spikes measured at baseline and after the magnets were turned on and off. C. Temporal response profile of all responding cells. Scale bar: $50 \mu \mathrm{m}$, ${ }^{*} p<0.005$, Repeated-measured ANOVA, Tukey's HSD. $n=13$ slices (total 4 animals). 


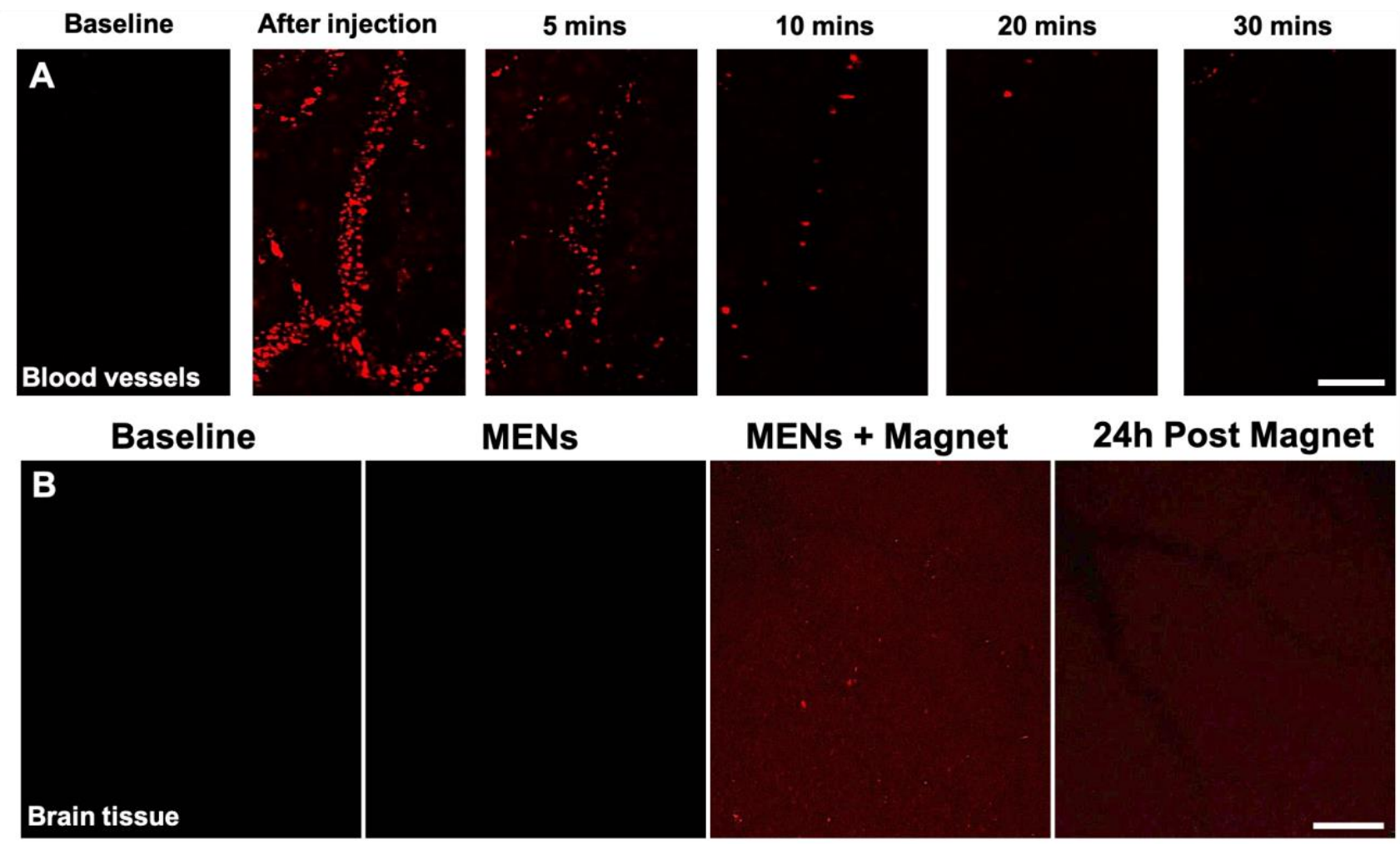

Figure 4. In vivo delivery of fMENs into mouse cortex. A. Vascular MENs level reduced significantly after 10 minutes post injection. In vivo two-photon images of cortical blood vessels were taken at baseline (B.L.) and at 5, 10, 20, and 30 minutes after intravenous injection of $10 \mu \mathrm{g}$ fluorescence labeled MENs (fMENs). No magnetic field was applied. Scale bar: $100 \mu \mathrm{m}$. B. In vivo two-photon images of layer 2/3 sensorimotor cortex were taken at baseline $(C 1)$ and after (C2) intravenous injection of $10 \mu \mathrm{g}$ fMENs, after applying 4000-5000 Oe magnetic field for 45 minutes (C3), and on the second day after injection (C4) indicates successful delivery of MENs to cortical tissue after magnet application, which MENs remained up to at least 24 hours after delivery. Scale bar: $100 \mu \mathrm{m}$. 

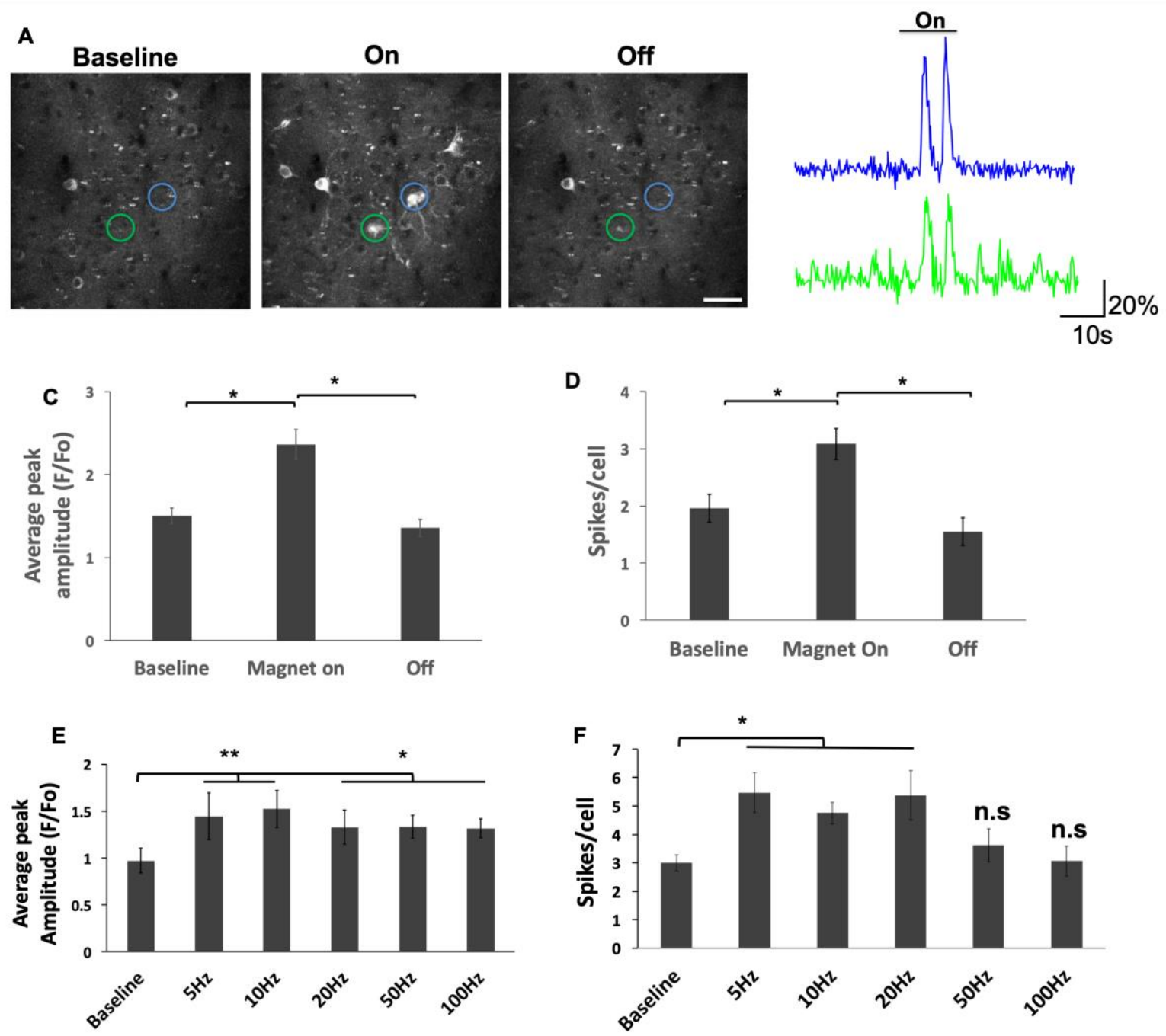

Figure 5. Magnetic stimulation activated cortical layer II/III neuron in vivo. A-B. Representative images $(A)$ and traces $(B)$ of calcium transients measured from layer II/III cortical neurons. C-D. Significant increases in average calcium spike amplitude (C) and mean spike number (D) of cortical neurons after magnet stimulation was turned on. $\mathrm{n}=5$ mice, ${ }^{*} \mathrm{p}<0.05$, one-way ANOVA, Tukey's HSD. Scale bar: $50 \mu \mathrm{m}$. Color of traces correspond to color of analyzed ROI. E-H. Increase in average calcium spike amplitude $(E, F)$ and total spikes per cell $(G, H)$ of cortical neurons when magnet was turned on at each frequencies of stimulations. Changes in the amplitudes and spike numbers of calcium transients at electromagnetic stimulation frequencies of $5,10,20,50$, and 100 $\mathrm{Hz}$. The data indicate that magnetic waves between $5-10 \mathrm{~Hz}$ were most effective in stimulating neurons in vivo. $\mathrm{n}=5,{ }^{\star} \mathrm{p}<0.05,{ }^{* *} \mathrm{p}<0.01$, two-way ANOVA, Tukey's HSD. Scale bar-100um 
A
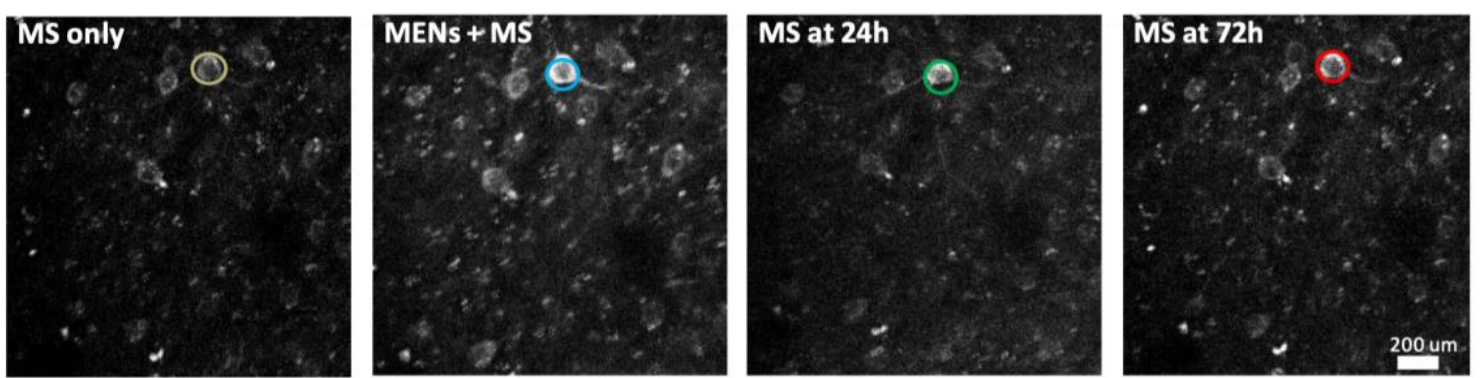

B

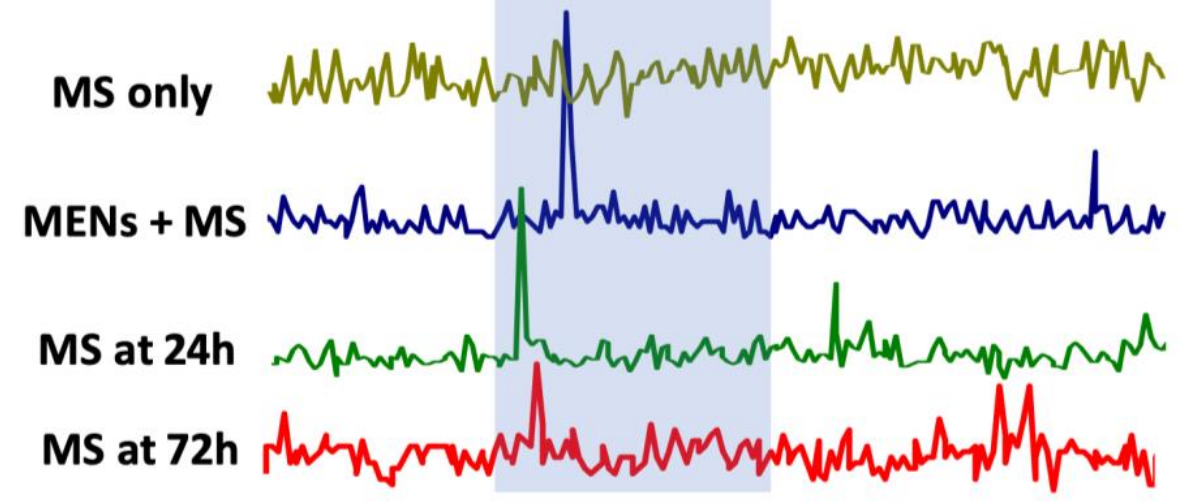

C

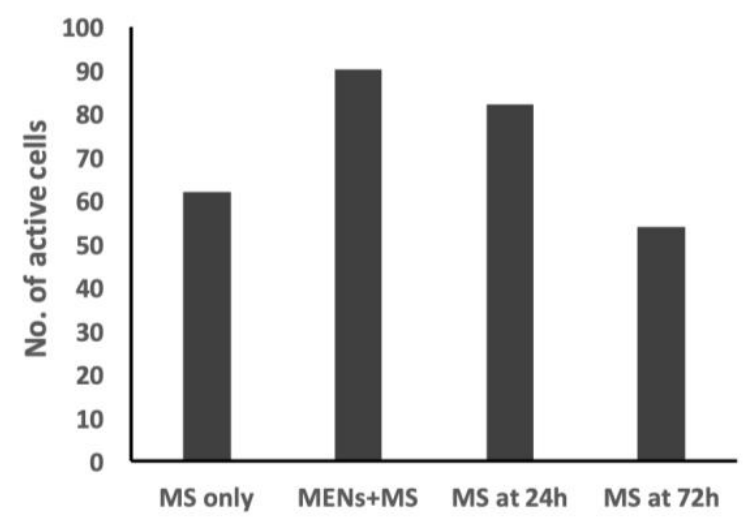

D

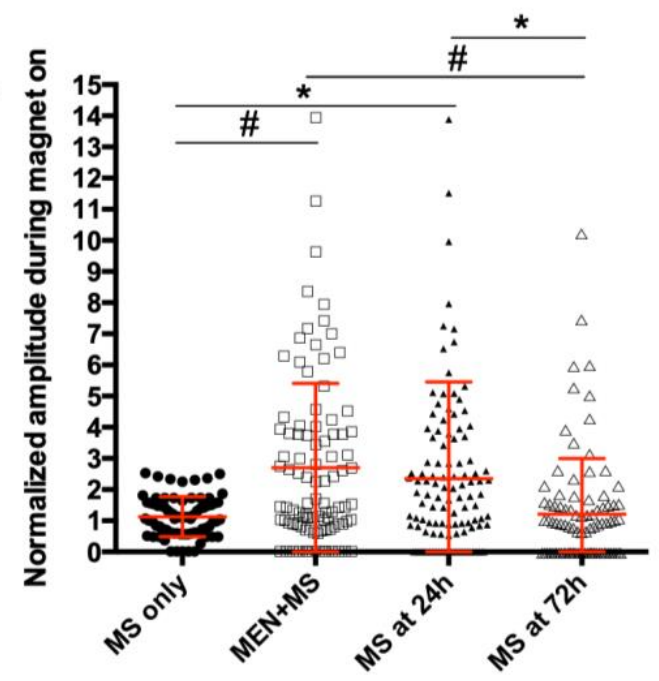


Figure 6. MENs-MS was effective in activating neurons in $\mathbf{2 4}$ hours after a single delivery of MENs. A-B. Representative two-photon images and traces of calcium signals of same cortical layer II/III neurons at baseline (MS only) and immediately after MENs delivery followed by MS (MEN+MS), MS at 24 hours after, and MS at 72 hours after. $10 \mathrm{~Hz}$ magnetic stimulation was applied for $20 \mathrm{~s}$ in the middle of recording period at each time point. C. There was an increase in number of active neurons after MENs-MS, which remained higher up to 24 hours after initial delivery. D. There were increases in response amplitude immediately after MENs-MS and at least 1 day after initial delivery, which returned to baseline level at 3 days post-MEN delivery, one-way ANOVA, Tukey's HSD, $n=5,{ }^{*} p<0.05, \# p<0.005$. 
A

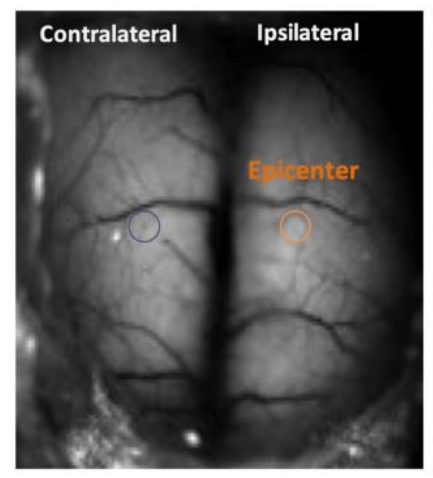

B

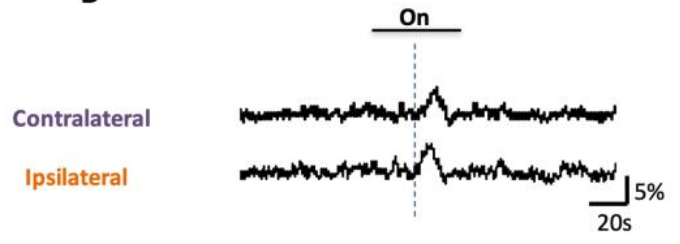

D

$+2 \mathrm{~mm}$

$+1 \mathrm{~mm}$

Contralateral

$-1 \mathrm{~mm}$

$-1 \mathrm{~mm}$

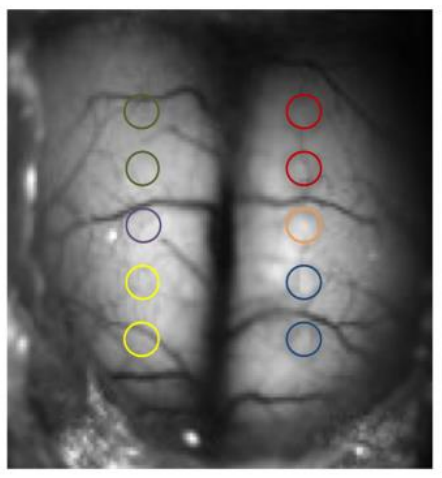

C
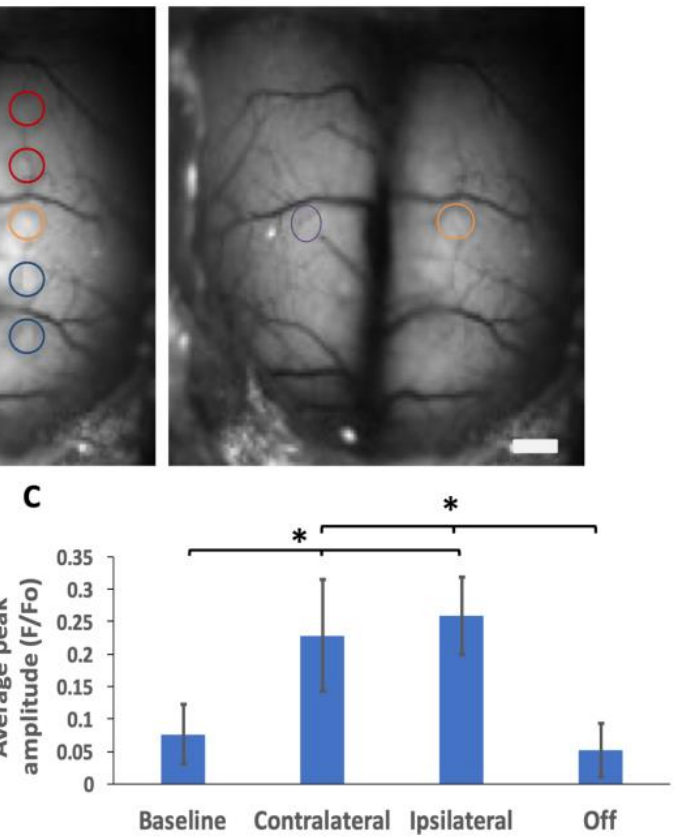

On

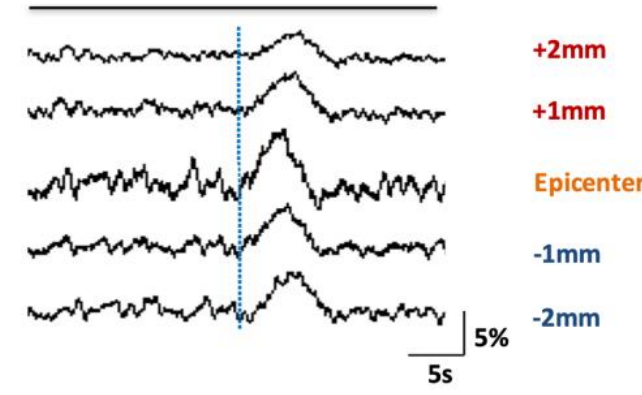

F

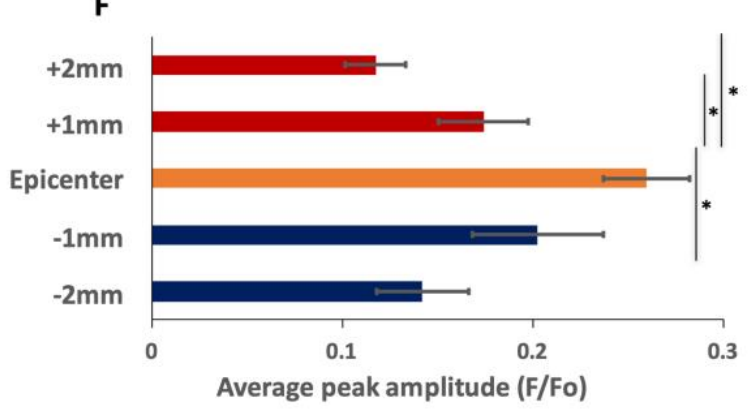

H

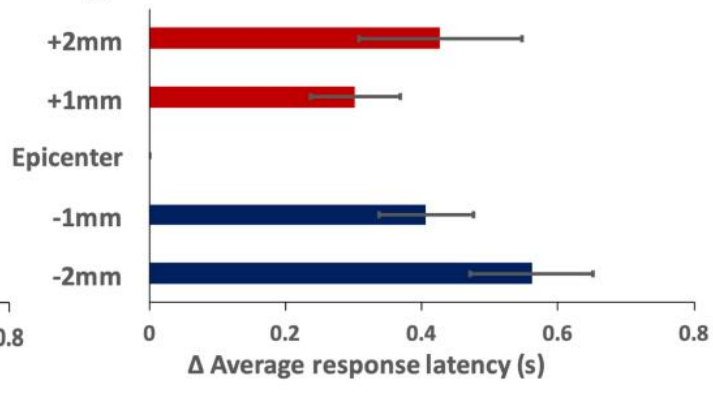


Figure 7. Mesoscopic activity imaging revealed initiation and spreading of MENsMS induced cortical activity in vivo. A-B. Representative images (A) and traces (B) of calcium transients measured from both hemispheres at baseline (left image) and after turning on and off magnetic stimulation (middle and right images). The epicenter was a cortical region where a conical magnet was applied to attract MENs. Dotted lines indicate the start of calcium spike. C. There were significantly higher amplitudes of calcium signals on cortex ipsilateral (at epicenter region) and contralateral (same region on opposite hemisphere) to MENs delivery induced by MS (10 Hz at 450 Oe for 30 seconds). $n=5,{ }^{*} p<0.05$, repeated-measured ANOVA, Tukey's HSD. D. Sample traces of calcium transients measured from epicenter, cortical regions anterior and posterior from epicenter, and areas of contralateral cortex. E, F. The mean amplitude of calcium signals in the epicenter was higher than that of cortical regions more anterior or posterior to it; the contralateral cortex had similar differences. G, $\mathbf{H}$. The latency period at the epicenter was the shortest than all other cortical regions. $n=5,{ }^{*} p<0.05$, one-way ANOVA, Tukey's HSD. Scale bar: $1 \mathrm{~mm}$. 


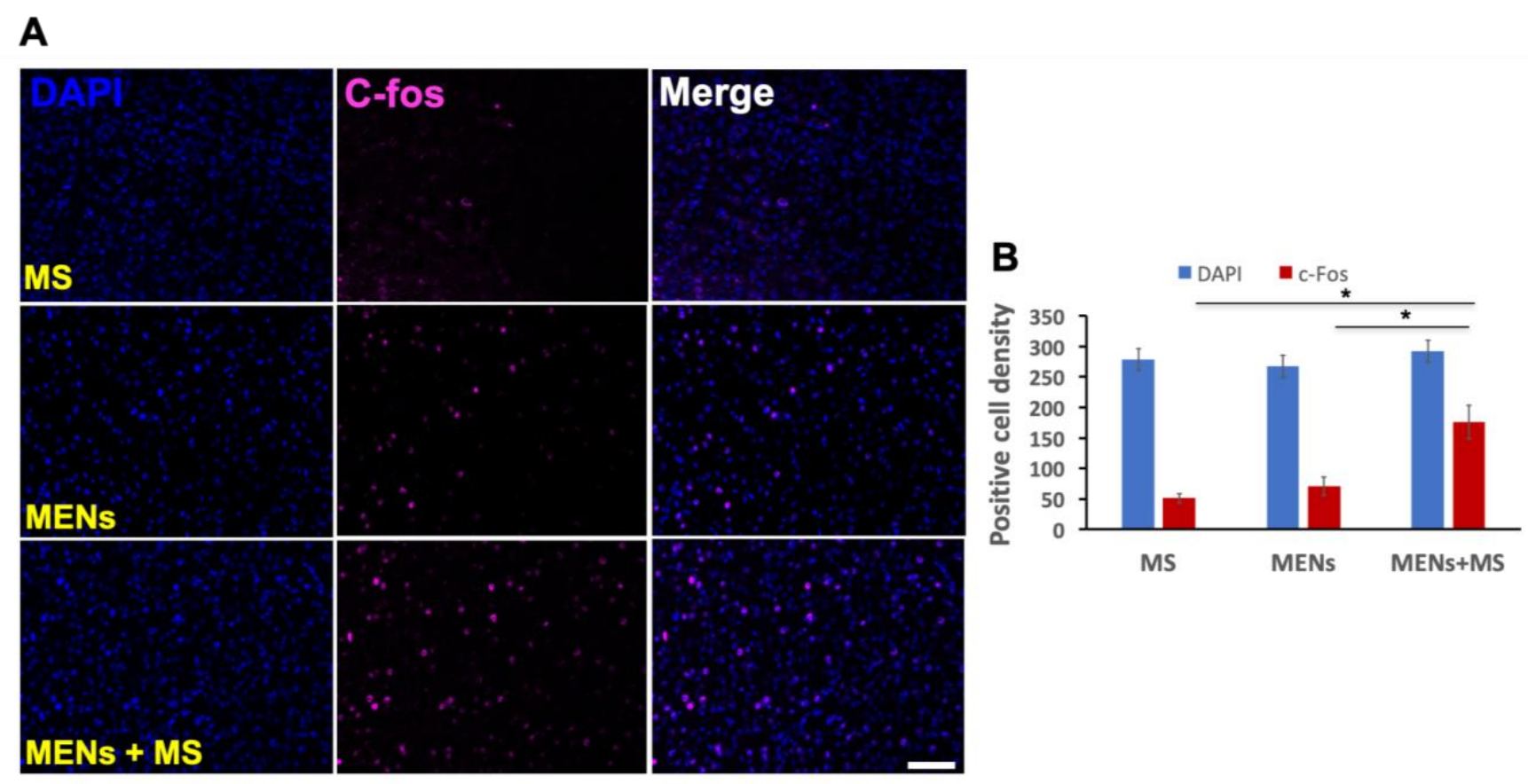

Figure 8. MENs-MS increased the number of $c$-fos expressing cells. A.

Representative images of immunofluorescence from mice that received magnetic stimulation (MS, top row), MENs delivery only (middle row), or MENs+MS (bottom row). Images were taken with an 20x objective. B. A significantly higher density of c-Fos expressing cells in MENs+MS group than either MS only or MENs only group. Scale bar: $20 \mu \mathrm{m} . \mathrm{n}=3$ mice/group, *: $\mathrm{p}<0.05$, Two-way ANOVA, Tukey's HSD. 

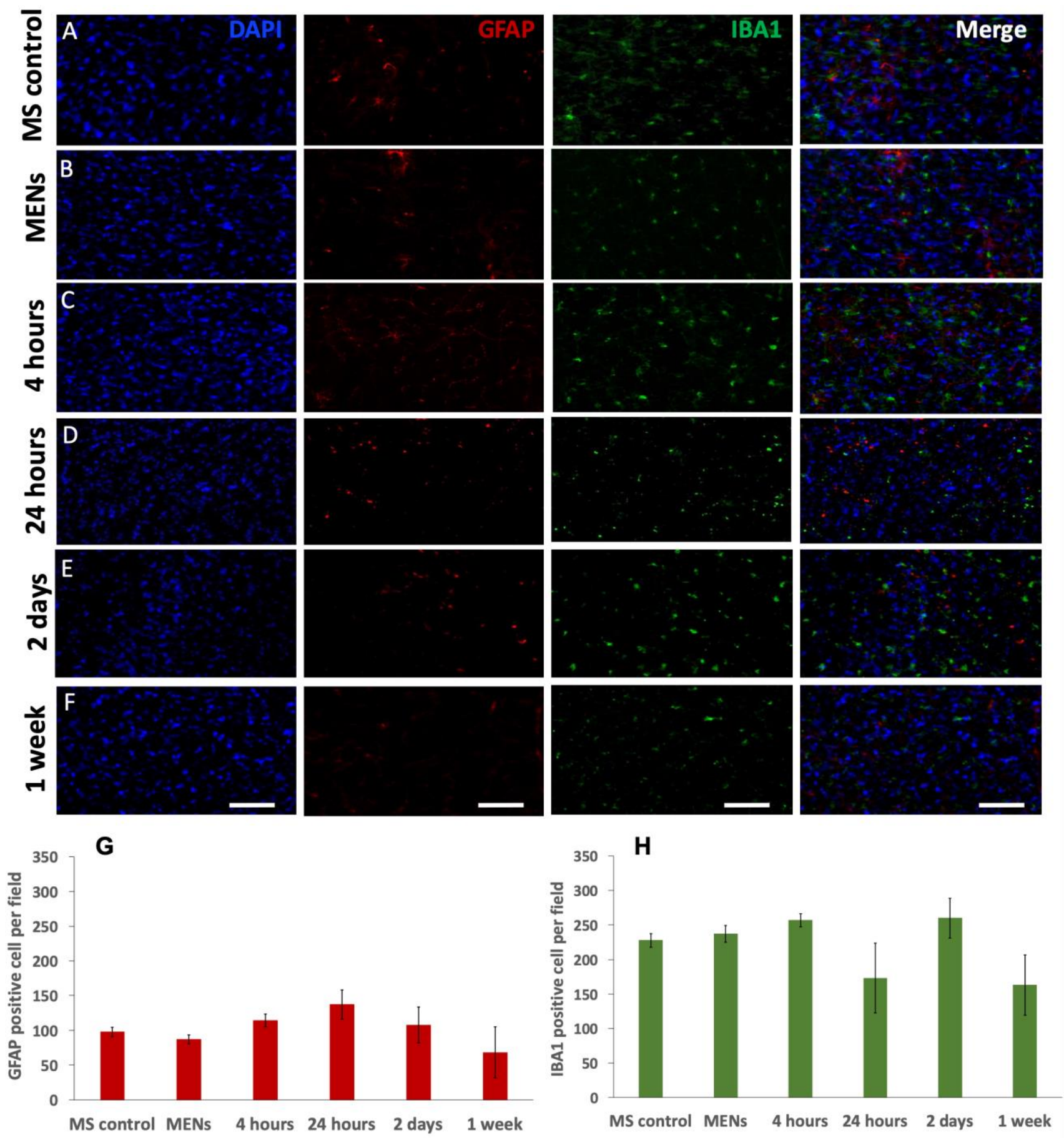
Figure 9. No significant changes in the numbers of GFAP and IBA1 positive cells after MEN delivery and MENs-MS. Brain sections were obtained from six groups of C57BL mice including MS control, MENs-delivery (MENs), and MENs delivery followed by MS at 4h, 24h, 2 days, and 1 week after. A-F. Sample confocal images of coronal cortical slices stained for DAPI, GFAP, and IBA1 at different time points. G, H. Quantifications of GFAP $(G)$ and IBA1 $(H)$ positive cells showed no significant differences in the numbers of these cells after MEN delivery or followed by MS. $n=4$ mice/group. Scale bar: $50 \mu \mathrm{m}$ 


\section{References}

1. Hummel Fea. Effects of non-invasive cortical stimulation on skilled motor function in chronic stroke. Brain 2005, 128: 164-174.

2. Miniussi Cea. Efficacy of repetitive transcranial magnetic stimulation/ transcranial direct current stimulation in cognitive neurorehabilitation. . Brain Stimulation 2008, 1: 326-336.

3. Demirtas-Tatlidede A, ..., Pascual-Leone A. Non-invasive brain stimulation in traumatic brain injury. Journal of Head trauma rehabilitation 2012, 27(4): 274-292.

4. Wagner T, Valero-Cabre, A., Pascual-Leone, A. Noninvasive human brain stimulation. Annual Review of Biomedical England 2007, 9: 527-565.

5. Sollmann N, Hauck, T., Tussis, L., Ille, S., Maurer, S., Boeckh-Behrens, T., Ringel, F., Meyer, B., Krieg, S.M. Results on the spatial resolution of repetitive transcranial magnetic stimulation for cortical language mapping during object naming in healthy subjects. BMC Neuroscience 2016, 17(67).

6. Sparing R, Mottaghy, F.M. . Noninvasive brain stimulation with transcranial magnetic or direct current stimulation (TMS/tDCS)-From insights into human memory to therapy of its dysfunction. Methods 2008, 44(f): 329-337.

7. Brunoni AR, Nitsche, M.A., Bolognini, N., Bikson, M., Wagner, T., Merabet, L., Edwards, D.J., Valero-Cabre, A., Rotenberg, A., Pascual-Leone, A., Ferrucci, R., Priori, A., Boggio, P.S., Fregni, F. Clinical research with transcranial direct current stimulation (tDCS): challenges and future directions. Brain Stimulation 2012, 5: 175-195.

8. Gradinaru V. TK, Zhang F., Mogri M., Kay K., Schneider B., Deisseroth K. Targeting and readout strategies for fast optical neural control in vitro and in vivo. Journal of Neuroscience 2007, 27(52): 14231-14238.

9. Fenno L, Yizhar, O., Deisseroth, K. . The development and application of Optogenetics. Annual Review of Neuroscience 2011, 34: 389-412.

10. Chen R, Romero G, Christiansen MG, Mohr A, Anikeeva P. Wireless magnetothermal deep brain stimulation. Science 2015, 347(6229): 1477-1480.

11. Huang H, Delikanli, S., Zeng, H., Ferkey, D.M., Pralle, A. Remote control of ion channels and neurons through magnetic-field heating of nanoparticles. Nature Nanotechnology 2010, 5(6): 602-606.

12. Bauer R, Martin, E., Haegele-Link, S., Kaegi, G., von Specht, M., Werner, B. . Noninvasive functional neurosurgery using transcranial MR imaging-guided focused ultrasound. Parkinsonism \& Related Disorders 2014, 20: 175-195.

13. Lipsman N, Schwartz, M. L., Huang, Y., Lee, L., Sankar, T., Chapman, M., et al. MRguided focused ultrasound thalamotomy for essential tremor: A proof-of-concept study. Lancet Neurology 2013, 12(5): 462-468. 
14. Bystritsky A, Korb, A. S., Douglas, P. K., Cohen, M. S., Melega, W. P., Mulgaonkar, A. P., et al. . A review of low-intensity focused ultrasound pulsation. Brain Stimulation 2011, 4(3): 125-136.

15. Yue K, Guduru R, Hong J, Liang P, Nair M, Khizroev S. Magneto-electric nano-particles for non-invasive brain stimulation. PloS one 2012, 7(9): e44040.

16. Guduru R, Liang P, Hong J, Rodzinski A, Hadjikhani A, Horstmyer J, et al. Magnetoelectric 'spin' on stimulating the brain. Nanomedicine (Lond) 2015, 10(13): 2051-2061.

17. Burgess E, Sylvester M, ..., Boggiana MM. Effects of Transcranial Direct Current Stimulations (tDCS) on Binge-eating disorder. International Journal of Eating Disorders 2016, 49(10): 930-936.

18. Guduru R. KS. Magnetic field-controlled release of paclitaxel drug from functionalized magnetoelectric nanoparticles. Particle \& Particle Systems Characterization 2014, 31: 605-611.

19. Silasi G. XD, Vanni M., Chen A, Murphy T. Intact skull chronic windows for mesoscopic wide-field imaging in awake mice. Journal of Neuroscience Methods 2016, 267: 141149.

20. Barson D, Hamodi, A.S., Shen, X. et al. Simultaneous mesoscopic and two-photon imaging of neuronal activity in cortical circuits. Nature Methods 2020, 17: 107-113.

21. Tsai-Wen Chen TJW, Yi Sun, Stefan R. Pulver, Sabine L. Renninger, Amy Baohan, Eric R. Schreiter, Rex A. Kerr, Michael B. Orger, Vivek Jayaraman, Loren L. Looger, Karel Svoboda, Douglas S. Kim. Ultrasensitive fluorescent proteins for imaging neuronal activity. Nature 2013, 499.

22. A. Kerlin MA, V. Berezovskii, R.C. Reid. Broadly tuned response properties of diverse inhibitory neuron subtypes in mouse visual cortex. Neuron 2010, 67(5): 858-871.

23. Bullitt E. Expression of C-fos-Like Protein as a marker for neuronal activity following noxious stimulation in Rats. Journal of Comparative Neurology 1990, 296: 517-530.

24. Imai Y. KS. Intracellular signaling in M-CSF-induced microglia activation: role of lba1. Glia 2002, 40: 164-174.

25. Liedtke W. EW, Bieri PL., Chiu FC., Cowan NJ., Kucherlapati R., Raine CS. GFAP is necessary for integrity of CNS white matter architecture and long-term maintenance of myelination. Neuron 1996, 17: 607-615.

26. Kaushilk $A, \ldots$, Nair M. Magnetically guided central nervous system delivery and toxicity evaluation of magneto-electric nanocarriers. Scientific Reports 2016, 6(25309).

27. Lankoff A. SW, Wegierek-Ciuk A., Lisowska H., Refsnes M., Sartowska B., Schwarze P., Meczynska-Wielgosz S., Wojewodzka M, Kruszewski M. The effect of agglomeration state of silver and titanium dioxide nanoparticles on cellular response of HepG2, A549 and THP-1 cells. . Toxicology Letters 2012, 208: 197-213. 
28. E F. The role of surface charge in cellular uptate and cytotoxicity of medical nanoparticles. International Journal of Nanomedicine 2012, 7: 5577-5591.

29. Okuda-Shimazaki J. TS, Kanehira K., Sonezaki S., Taniguchi A. Effects of Titanium Dioxide nanoparticle aggregate size on gene expression. International Journal of Molecular Science 2010, 11: 2383-2392.

30. Nair M, Guduru R., Liang P., Hong J., Sagar V., Khizroev S. Externally ocntrolled ondemand release of anti-HIV drug using magneto-electric nanoparticles as carriers. Nature Communications 2013, 4(1707).

31. Dilnawaz F. SA, Mewar S., Sharma U., Jagannathan N.R., Sahoo S. The transport of non-surfactant based paclitaxel loaded magnetic nanoparticles across the blood brain barrier in a rat model. Biomaterials 2012, 33(33): 2936-2951.

32. Kim JS, Yoon $\mathrm{T}, \ldots$, Cho $\mathrm{MH}$. Toxicity and tissue distribution of magnetic nanoparticles in mice. Toxicological Sciences 2006, 89(1): 338-347.

33. Wu B. YA. Quercetin inhibits c-fos, Heat Shock Protein, and Glial fibrillary acidic protein expression in injured astrocytes. Journal of Neuroscience Research 2000, 62: 730-736.

34. Groves A. KY, Jonnalagadda D., Rivera R., Kennedy G., Mayford M., Chun J. A functionally defined in vivo astrocyte population identified by c-Fos activation in a mouse model of multiple sclerosis modulated by S1P signaling: immediate-early astrocytes (ieAstrocytes). eNeuro 2018.

35. Koziara JM LP, Allen DD, Mumper RJ. The blood-brain barrier and brain drug delivery. Journal of Nanoscience Nanotechnology 2006, 6: 2712-2735.

36. Y. J. Yu YZ, M. Kenrick, K. Hoyte, W. Luk, Y. Lu, J. Atwal, J. M. Elliott, S. Prabhu, R. J. Watts, M. S. Dennis. . Boosting brain uptake of a therapeutic antibody by reducing its affinity for a transcytosis target. Science Translational Medicine 2011, 3.

37. Qiao R. JQ, Huwel S., Xia R., Liu T., Gao F., Galla H., Gao M. Receptor-mediated delivery of magnetic nanoparticles across the blood-brain barrier. ACS Nano 2012, 6(4): 3304-3310.

38. Chu C. JA, Lesniak W., Thomas A., Lan X., Linville R. et al. Optimization of osmotic blood-brain barrier opening to enable intravital microscopy studies on drug delivery in mouse cortex. Journal of Controlled Release 2020, 317: 312-321.

39. Zhang Q. FB, Zhang Z. . Borneol, a novel agent that improves central nervous system drug delivery by enhancing blood-brain barrier permeability. Drug Delivery 2017, 24(1): 1037-1044. 